\title{
FROM STRUCTURE TO STRUCTURAL DYNAMICS
}

M. Chergui, Laboratoire de Spectroscopie Ultrarapide, Ecole Polytechnique Fédérale de Lausanne, ISIC, FSB, Station 6, $\mathrm{CH}-1015$ Lausanne, Switzerland

The atomic scale resolution of space (the Ångström) was achieved a century ago with the discovery of X-ray and electron diffraction. Already then, the notion of nuclear motion and disorder was considered by P. Debye. It would take decades before the idea of driving chemical reaction by light and capturing the new structures by diffraction would become possible thanks to Phil Coppens' pioneering works. With the advent of synchrotrons and more recently X-ray free electron lasers, we have moved into the era of ultrafast (picosecond to femtosecond) probing of (bio)chemical reactions and phase transitions in materials. In this talk, I will review some of the landmarks leading to the development of the field of ultrafast structural dynamics using X-ray pulses and will then present some recent results from the literature and from our work on chemical and biological systems and on solid materials. 